\title{
Eclampsia complicated with pulmonary edema and postpartum cardiomyopathy - a challenging task
}

\begin{abstract}
Peripartum Cardiomyopathy (PPCM) is a disease that affects women in their last months of pregnancy to the first month after delivery. It is a relatively rare disease, which can have devastating consequences and should be promptly identified and appropriately treated. Its diagnosis is a challenging task as it is often delayed because its symptoms closely resemble those of normal pregnancy and postpartum period. The disorder carries a high mortality rate, therefore requires vigilance. The aim of present case report is to describe the presentation and management of a case of PPCM so as to develop awareness because overall prognosis is good in majority of the cases if identified early.
\end{abstract}

Keywords: eclampsia, pulmonary edema, postpartum cardiomyopathy
Volume 2 Issue 4 - 2017

\author{
Sonali Somani,' Shashikant Somani, ${ }^{2}$ Sunita \\ Sudhir,'S Meerajuddin² \\ 'Department of Obstetrics \& Gynaecology, Kamineni Institutes \\ of Medical Sciences, India \\ ${ }^{2}$ Department of Anaesthesiology, Kamineni Institutes of Medical \\ Sciences, India
}

\begin{abstract}
Correspondence: Shashikant Somani, Department of Anaesthesiology, Kamineni Institutes of Medical Sciences, Narketpally, Aditya Arcade, Sri Sai Block - C, I-8-678/ 20 Flat No.303, Electric Substation, VST Signal, Opp. Dundoo Oil Mill, Azambad, Hydrabad, Telangana, India, Tel 0944I365I65, Email drsgsomani@gmail.com
\end{abstract}

Received: April 12, 2017 | Published: June 01, 2017

\section{Introduction}

Peripartum Cardiomyopathy (PPCM) is a life-threatening disorder. Its incidence varies from $0.2 \%$ to $3 \%{ }^{1-3}$ Risk factors include multiparty, advanced maternal age, obesity, gestational hypertension, pre-eclampsia, eclampsia, diabetes, malnutrition etc. ${ }^{4,5}$ Current diagnosis of PPCM is based on presence of 4 clinical criteria:

i. Cardiac failure in last month of pregnancy or within 5 months of delivery.

ii. Absence of identifiable cause of cardiac failure.

iii. No features of heart disease prior to the last month of pregnancy.

iv. Left ventricle ejection fraction of less than $45 \%{ }^{6,7}$

Currently, it is considered a separate, distinct disease from Cardiomyopathy due to the lack of preexisting cardiac issues. ${ }^{8,9}$ Since most signs and symptoms of late pregnancy mimic that of cardiac failure, the diagnosis of PPCM is often more difficult and delayed. Therefore, disorder is under recognized, but it has devastating consequences with Mortality as high as $20 \%$ to $50 \%{ }^{10}$ The purpose of present case presentation is to create awareness regarding early diagnosis and prompt management, so as to reduce maternal mortality.

\section{Case report}

A 21 year-old, primigravida with 8 months of amenorrhea brought to labor room Kamineni Institutes of Medical Sciences with h/o of convulsions at home. Patient was apparently asymptomatic 6 hours before, then suddenly she developed headache and blurring of vision followed by two episodes of convulsions which were generalized tonic colonic, each lasted for 2-3 minutes associated with frothing, tongue bite $\&$ involuntary maturation. There was $\mathrm{h} / \mathrm{o}$ of pain abdomen on \& off since 3 days, No h/o bleeding or leaking per vaginum. First two trimesters were uneventful; her medical, surgical, family \& personal history was noncontributory.

On physical examination, she was drowsy, irritable, responding to painful stimuli, not well oriented. B/L pupils normal size reacting to light. No pallor/icterus/cyanosis/ lymphadenopathy. There was Grade 2 edema in lower extremities. Thyroid, breast, spine were normal.

She was afebrile, pulse rate of $-110 / \mathrm{min}$, normal in volume, regular in rhythm. Blood pressure of 160/100 $\mathrm{mm}$ of $\mathrm{Hg}$, in right arm, supine position. Respiratory rate of 24 cycles/min. Systemic examinationRS- normal vesicular breath sounds no crepts/rhochi. CVS- S1, S2 heard, no murmur. CNS-No gross focal neuro deficit, Deep tendon reflexes exaggerated.

\section{Obstetric examination}

Fundal Height-uterus 32-34wks \& Symphy so fundal height-32cm. Single live intrauterine fetus in cephalic presentation with clinically very less liquor, Fetal HR was 110/min with Non reactive NST and contractions were every 2-4 minutes, lasting for 20-25seconds.

\section{P/V examination}

Cervix soft midpostion, $50 \%$ effaced, so admitting 1 finger, presenting part vertex high up with adequate pelvis in early labour.

Immediate treatment-Airway secured with airway and suctioning was done.O2 inhalation (5lt/min) was given. Two IV canulation with 18 No. canula was done. IV fluids started, patient was positioned on left lateral position, Inj MgSO4 loading dose 14gm (4gm IV/ 10gms IM) given. Cap depin $5 \mathrm{mg} \mathrm{S} / \mathrm{L}$ given, Foleys cauterization was done. High risk consent was taken.

Pregnancy induced hypertension (PIH) profile showed: hemoglobin-13.8g/dL; hematocrit-30\%; platelets-2 lakhs $/ \mathrm{mm}^{3}$; Prothrombin time-12.5 seconds INR-1.0; partial thromboplastin time28 seconds; bleeding time, clotting time, serum electrolytes were within normal limits. Renal profile- serum urea- $17 \mathrm{mg} / \mathrm{dL}$; serum creatinine- $0.7 \mathrm{mg} / \mathrm{dL}$; uric acid- $6.4 \mathrm{mg} / \mathrm{dL}$. Liver function test-within normal limit. Random blood sugar- $82 \mathrm{mg} / \mathrm{dl}$. Urine analysis revealed $4+$ albumin \& 6-8 pus cells. Blood grouping-O positive Serology HIV, HBsAg, VDRL - non reactive. Fundoscopy -Normal. Obstetric 
scan showed Single live intrauterine fetus in cephalic presentation 33 week, BPD- 6.5cms; Femur length $-5.8 \mathrm{cms}$; Estimated Fetal Weight $-1.9 \mathrm{~kg}$, placenta-anterior, upper segment; AFI-4cms Suggestive of Severe oligohydramnios.

Pre-anesthetic evaluation was done. Blood \& FFP's were reserved. Patient was taken for emergency lower segment cesarean section under general anesthesia. She delivered a live male baby of wt $2 \mathrm{~kg}$ with APGAR $8 \& 10$ at $1 \& 5 \mathrm{~min}$ respectively. Placenta revealed normal morphology. Uterus well retracted. Patient was extubated. In post-op period, after 4 hours, patient started complaining of shortness of breath which was worsened within 30minute. There was labored RR of $34 / \mathrm{min}, \mathrm{SpO} 2$ dropping to $85 \%$, PR-150/min, BP of $160 / 104$ $\mathrm{mm}$ of $\mathrm{Hg}$. RS- revealed bilateral creptations more at the basal region. In view of pulmonary edema, patient was put on mechanical ventilator (CMV with PEEP). Labetalol (10mg) IV was given to control severe hypertension. X ray chest: showed pulmonary edema (Figure 1).

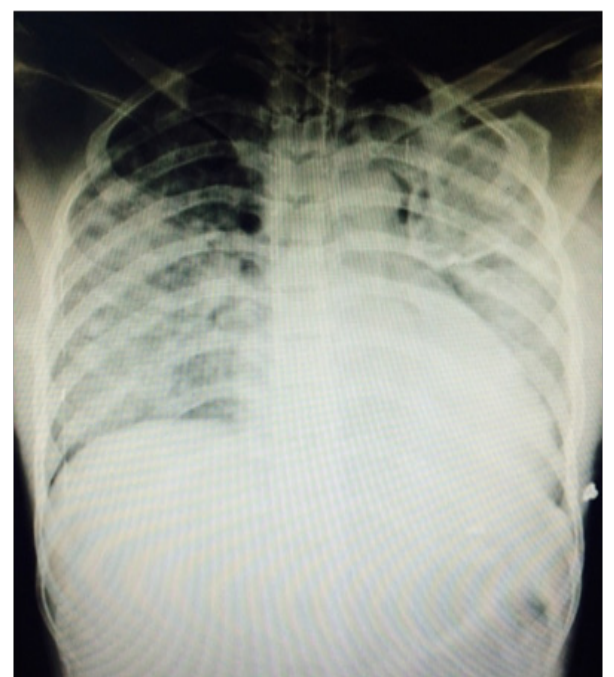

Figure I Electro cardiogram -Sinus tachycardia \& occasional ventricular ectopic. Acute left ventricular dysfunction, Global hyperkinesias \& EF- < 30\%.

\section{Arterial blood gas analysis}

Revealed an uncompensated respiratory \& metabolic acidosis with hypoxemia (pH-7.17, 38mmHg, PaCo2-36mmHg, SpO2-85\%, $\mathrm{Hco} 3-13.1 \mathrm{mEq} / \mathrm{L}$ ). The patient was diagnosed with Postpartum Cardiomyopathy based on clinical symptoms of congestive heart failure and pulmonary edema in absence of other cause. During a 4-day stay in Acute Obstetric Unit, patient was on mechanical ventilator. Medical treatment given was Inj dobutamine $5 \mu \mathrm{gm} / \mathrm{kg} / \mathrm{min}$ infusions which was tapered; Inj furosemide $20 \mathrm{mg}$ IV BD; IV fluids according to CVP and urine output, broad spectrum antibiotic covering aerobic $\&$ anaerobic organism. Strict I/O charts \& vitals monitoring done. On 4th post op day she was extricated. Post-operative day 5, 6, 7 were uneventful. Suture removal was done on 7 th post operative day. She was actively ambulated, encourgaed for breast feeding. She was counseled about the risks associated with subsequent pregnancies \& discharged on 9 th post operative day.

\section{Discussion}

Preeclampsia is a multiorgan disease, characterized by hypertension $\&$ proteinuria, at or after 20 weeks' of gestation. It is considered to be severe when BP is $>160 \mathrm{mmHg}$ systolic and $110 \mathrm{mmHg}$ diastolic, signs and symptoms are consistent with end organ damage like neurological symptoms, such as headache or visual disturbances, impaired coagulation, renal, hepatic function \& pulmonary edema. ${ }^{7}$ Pulmonary edema, ascites, and gestational proteinuria have been attributed to capillary leak syndrome. ${ }^{6}$ Eclampsia is severe preeclampsia complicated with convulsion. In present case, diagnosis of PPCM was difficult because some patients with preeclampsia \& eclampsia may presents with dyspnea, fatigue \& pedal edema. Furthermore a history of preeclampsia is frequently reported in cases of PPCM.

\section{Pathogenesis}

The etiology and pathogenesis seems to be multifactorial \& poorly understood. Potential causes have been proposed, however, no supporting evidence has been discovered. ${ }^{11,12}$ It is speculated that the absence of immune response during and immediately following pregnancy allows for undetected viruses to enter and replicate in the body. ${ }^{10}$ The presence of a low-grade chronic inflammatory process results in the release of endotoxin and pro-inflammatory cytokines. ${ }^{13}$ These molecular markers of an inflammatory process are found in most of the patients. $90 \%$ of the patients show high levels of plasma C-reactive protein that correlates positively with LV end-diastolic and end-systolic dimensions and inversely with LV ejection fractions. ${ }^{14} \mathrm{~A}$ hereditary predisposition is also suggested and strong consideration should be given to screening family members. ${ }^{15}$ Additionally, oxidative stress, which is thought to contribute to or stimulate the pathophysiologic associated with preeclampsia, is thought to be a key component in the development of PPCM. ${ }^{4}$

\section{Clinical presentation}

Early signs of PPCM mimic normal experiences during late stage pregnancy, which results in a delayed diagnosis. ${ }^{16}$ A comprehensive examination including laboratory and radiology investigation reveal cardiomegaly, tachycardia, arrhythmias, left ventricular hypertrophy, premature ventricular contractions and bundle branch block. ${ }^{17,18}$ The diagnosis is based on exclusion.

\section{Management}

Treatment should be goal directed and focused on optimizing hemodynamic, relief of symptoms and treatment of precipitating factors. Our aim is to reduce the after load and preload and increase the overall contractility. There is no specific treatment plan for PPCM. In general, it is similar to other forms of heart failure. Medications commonly used include digitalis, diuretics-judicious use of diuretics to relieve pulmonary edema, after load reduction to decrease the work of the heart by controlling hypertension. ${ }^{19,20} \beta$ blockers, ACEinhibitors, vasodilators, spironolactone, anticoagulants therapy should be considered in view of the low left ventricular EF, which predisposes to thrombus formation, especially in the peripartum period which is a hypercoagulable state and antiarrhythmics. ${ }^{19-21}$ In patients with critical hemodynamic state with cardiogenic shock, hemodynamic support with pressers should be considered. Patients are also encouraged to implement a dietary treatment approach, which includes restrictions on daily salt intake. Other drugs like immunosuppressive are under evaluation.

\section{Prognosis}

Overall prognosis of PPCM is good in majority of the cases, although some patients may progress to irreversible heart failure. 


\section{Future outcome}

A subsequent pregnancy carries a high risk of relapse, significant decrease of left ventricular function and mortality of approximately $55 \%$ They require appropriate medical management and a multidisciplinary approach throughout pregnancy and delivery to improve maternal \& fetal outcome..$^{22,23}$ There is no consensus regarding recommendations for future pregnancy after PCM but patients whose left ventricular size or function does not return to normal should be counseled strongly to avoid subsequent pregnancy. ${ }^{1}$

\section{Conclusion}

PPCM is a disease that affects women in their last months of pregnancy to the first month after delivery. It has a multifactorial origin. It is a relatively rare disease, which can have divesting consequences and should be promptly identified and correctly treated. If PPCM is suspected, a multidisciplinary team that includes Anesthesiologist, Obstetricians, Cardiologists and Critical care specialists will be needed to help in diagnosis and management. It is diagnosed through exclusion. Diagnosis of PPCM is challenging and requires vigilance. Management should begin with more targeted therapies. Long-term prognosis is favorable when ventricular size and contractility returns to normalcy. Effective treatment reduces mortality rates and increases the chance of complete recovery of ventricular systolic function.

\section{Acknowledgements}

None.

\section{Conflict of interest}

Author declares that there is no conflict of interest.

\section{References}

1. Pearson GD, Veille JC, Rahimtoola S, et al. Peripartum cardiomyopathy: National Heart, Lung and Blood Institute and Office of Rare Diseases Workshop recommendations and reviews. JAMA. 2000;283(9):1183-1188.

2. Fett JD, Christie LG, Carraway RD, et al. Five-year prospective study of the incidence and prognosis of peripartum cardiomiopathy at a single insitution. Mayo Clin Proc. 2005;80(12):1602-1606.

3. Brar SS, Khan SS, Sandhu GK, et al. Incidence, mortality and racial differences in peripartum cardiomyopathy. $\mathrm{Am} J$ Cardiol. 2007;100(2):302-304.

4. Selle T, Renger I, Labidi S, et al. Reviewing peripartum cardiomyopathy: current state of knowledge. Future Cardiol. 2009;5(2):175-189.

5. Ansari AA1, Fett JD, Carraway RE, et al. Autoimmune mechanism as the basis for human peripartum cardiomyopathy. Clin Rev Allergy Immunol. 2002;23(3):301-324.
6. Sibai BM, Stella CL. Diagnosis and management of atypical preeclampsia-eclampsia. Am J Obstet Gynecol. 2009;200(5):481e1-481e7.

7. Polley LS. Hypertensive disorders. In: Chestnut DH, Polley LS, et al. editors. Chestnut's Obstetric Anesthesia: Principles and Practice. 4th ed Philadelphia, Pennsylvania; 2009. p. 975-1003.

8. Karaye K, Henein M. Peripartum cardiomyopathy: A review article. Int J Cardiol. 2013;164(1):33-38.

9. De Ceuninck M, Vermeulen J, Vrints C. Peripartum cardiomyopathy: A spoiled birthday party. Acta Cardiol. 2003;58:367-370.

10. Abboud J, Murad Y, Chen-Scarabelli C, et al. Peripartum cardiomyopathy: a comprehensive review. Int J Cardiol. 2007;118(3):295-303.

11. Bhattacharyya A, Basra SS, Sen P, et al. Peripartum Cardiomyopathy: a review. Tex Heart Inst J. 2012;39(1):8-16.

12. Blauwet L, Sliwa K. Peripartum cardiomyopathy. Obstet Med. 2011;4(2):44-52.

13. Rauchhaus M, Coats AJ, Anker SD. The endotoxin-lipoprotein hypotesis. Lancet. 2000;356(9233):930-933

14. Sliwa K, Förster O, Libhaber E, et al. Peripartum cardiomyopathy: inflammatory markers as predictors of outcome in 100 prospectively studied patients. Eur Heart J. 2006;27(4):441-446.

15. Fett JD. Peripartum cardiomyopathy in both surrogate and biological mother. Hum Reprod. 2005;20(9):2666-2668.

16. Sliwa K, Hilfiker-Kleiner D, Petrie MC, et al. Current state of knowledge on aetiology, diagnosis, management, and therapy of peripartum cardiomyopathy: A position statement from the heart failure association of the European society of cardiology working group on peripartum cardiomyopathy. Eur J Heart Fail. 2010;12(8):767-778.

17. Sean Martin, Daniel Short, Chih Mun Wong, et al. A change of heart: Case series of peripartum cardiomyopathy. Case Rep Obstet Gynecol. 2013;2013:563158.

18. Twomley KM, Wells GL. Peripartum cardiomyopathy: A current review. Journal of Pregnancy. 2010;2010:149127.

19. GivertzM.Peripartum cardiomyopathy. Circulation. 2013;127:622-626.

20. Bailey D. Peripartum cardiomyopathy. Anaesth Intensive Care 1992;20:397-398.

21. Zhanje F. Peripartum cardiomyopathy. N Z Med J. 2013;126:85-90.

22. Shannon-Cain J, Hunt E, Cain BS. Multidisciplinary management of peripartum cardiomyopathy during repeat cesarean delivery: a case report. AANA J. 2008;76(6):443-447.

23. Mishra TK, Swain S, Routray SN. Peripartum cardiomyopathy. J Assoc Physicians India. 2006;61(4):268-273. 